\title{
THE RISK MODEL OF DEVELOPING SCHIZOPHRENIA BASED ON TEMPERAMENT AND CHARACTER
}

\author{
Mirjana Miskovic ${ }^{1}$, Dragan Ravanic ${ }^{2}$, Dragic Bankovic ${ }^{3}$, Nera Zivlak-Radulovic ${ }^{1}$, \\ Visnja Banjac $^{1}$ \& Tatjana Dragisic ${ }^{1}$ \\ ${ }^{1}$ Clinic of psychiatry, University Clinical Center of the Republic of Srpska, Banjaluka, Bosnia and Herzegovina \\ ${ }^{2}$ University of Kragujevac, Faculty of Medical Sciences, Kragujevac, Serbia \\ ${ }^{3}$ University of Kragujevac, Faculty of Natural Sciences and Mathematics, Kragujevac, Serbia
}

received: 5.9.2017;

revised: 29.12.2017;

accepted: 9.1.2018

\section{SUMMARY}

Introduction: Cloninger's psychological model of temperament and character confirms that the personality development is influenced by biological and psychological processes. The aim of this study is to examine personality dimensions and to determine which variable separates the healthy from the ill in the best way and could be a possible psychological marker for the presence of the illness.

Methods: This research included 152 subjects, 76 patients with schizophrenia and 76 healthy controls, selected on the basis of medical interviews, random population sampling model from a wider social community using the independent T-Tests. The Temperament and Character Inventory (TCI), which compared personality traits of the patients with schizophrenia and the healthy control group, was used. Dependence of variables in these categories was assessed using the Chi-square and Fisher's tests, and the impact of variables on schizophrenia was tested using univariate and multivariate binary logistic regression. The same method was used for making the mathematical model.

Results: Unlike the control group, patients with schizophrenia exhibited higher Harm avoidance (HA) and Self - transcendence (ST) scores as well as lower Self - directedness (SD) and Cooperativeness (C) scores. Multivariate binary logistic regression showed that Responsibility, Purposefulness, Resourcefulness, Cooperativeness and Compassion dimensions were significantly more present in the patients with schizophrenia. The new variable Model (area $=0.896, p<0.0005)$ is composed of five TCI parameters. It proved to be a reliable marker for separation the healthy from the ill ones (area=0.896, $p<0.0005)$. It has a good sensitivity (80\%) and specificity (84\%).

Conclusions: Research has emphasized variables in the temperament and character inventory, which are the best markers for distinguishing between the healthy and the ill, thus making the mathematical model.

Key words: schizofrenia - TCI - temperament - character

$$
* * * * *
$$

\section{INTRODUCTION}

Schizophrenia is an illness that represents a paradigm of psychiatry - mental disorder that takes the central topic for the psychiatric science and practice. Therefore, for more than a century, schizophrenia is a constant challenge for researchers and psychiatric clinicians. The fact that schizophrenia is exclusively a human illness gives an additional human dimension to all the efforts in the research and observation of the rich psychopathology (Popovic 2014). The premorbid and prodromal stages of schizophrenia are usually examined retrogradely, after the diagnosis of clear symptoms of the illness. The question is whether there are any specific personality traits, respectively premorbid signs and symptoms preceding the disorder, which are regarded as an integral part of the disorder (Ostojic 2012). The prodromal stage usually occurs in adolescence and early twenties. A clinical picture of schizophrenia is usually determined by the onset of the prodromal stage, lasting up to several years, and can be manifested through gradual social withdrawal (Schenkel \& Silverstein 2004). The prodromal stage, lasting up to several years, is taken into consideration only if it is followed by clear symptoms and signs of the schizophrenic process. Robert Cloninger's psychological personality model (Cloninger et al. 1993, Cloninger et al. 1994) presents a seven - dimension personality model through which the author is trying to include and describe universal individual differences. The Model describes the structure and development of a personality as a complex system consisting of the interactive domains of temperament and character. Cloninger designed this personality model so that it includes the four dimensions of temperament (Novelty Seeking - NS, Harm Avoidance - HA, Reward Dependence - RD, Persistence - P) and three dimensions of character (Self-directedness - SD, Cooperativeness $-\mathrm{C}$, Self-transcendence $-\mathrm{ST}$ ), whose specific combinations determine the personality type, as well as the presence and type of a disorder. Tridimensional personality questionnaire (TPQ) is the older test on personality traits than TCI is, and it measures three characteristics of temperament (NS, HA and RD) (Cloninger 1987). We used the TCI questionnaire, in order to compare the seven dimensions of personality between the patients with schizophrenia and the control group. Results of the research conducted in Japan using the TCI questionnaire, between the patients with schizo- 
phrenia and the control group of healthy subjects, showed that there were differences in the personality traits between these two groups (Ohi et al. 2012). Studies using this model showed that personality configuration described in the patients with schizophrenia is as follows: patients exhibited high Harm Avoidance score and low Novelty Seeking, Reward Dependence, Self-directedness, Cooperativeness and Self-transcendence scores (Ritsner et al. 2003, Kurs et al. 2005, Hori et al. 2008, Aukst Margetic et al. 2009). High Harm Avoidance score is mentioned as a marker of predisposition to schizophrenia (Ritsner et al. 2003). It is a question whether some variable from the TCI may (by itself) be a marker of the illness. The aim of our study is to examine personality dimensions and to determine which variable separates the healthy from the ill ones in the best way, thus being a possible psychological marker which would contribute to the illness.

\section{METHOD}

The sample consisted of 152 subjects. The study included 76 patients with schizophrenia over the age of 18 , of both genders, who met the eligibility criteria ( $F$ 20) defined by the Tenth revision of International Statistical Classification of Diseases and Related Health Problems - ICD, hospitalized or infirmary treated patients at the Psychiatry Clinic of the University Clinical Center of the Republic of Srpska during 2015. Excluding criteria for the research were other psychiatric disorders, chronic neurological, internistic and other non-psychiatric illnesses in personal history. After that, the testing using the PANSS scales (negative, positive and general psychopathology scales were used) was conducted, in order to determine the current symptoms of schizophrenia, and then followed the BPRS scale and the TCI questionnaire. There were 76 healthy controls in the control group ( 35 males and 41 females), selected on the basis of medical interviews, random sampling model, population sampling from a wider social community, aged 18-65 years. Before the research, internal disorders and psychiatric disorders were excluded, as well as the use of medications. After the participants gave their oral consent, they were informed of the purpose of the study and guaranteed the right to anonymity prior to administering the questionnaire. A written informed consent was obtained from the participants prior to the onset of the study. For the purposes of testing, a classic clinical psychiatric exploration form was used, which implied a structured clinical interview for the assessment of schizophrenia diagnosis according to the criteria.

The study was carried out as an academic and nonprofit research, relying on the principles of the Good Clinical Practice and Declaration of Helsinki. It was designed as a controlled, non treatment, open and prospective research. The study was approved by the Ethics Committee to the University Clinical Center of the Republic of Srpska. The following assessment tools were used:
- The Temperament and Character Inventory (Cloninger et al. 1993) is used to assess the TCI traits. It was designed as a modified version of the first Tridimensional Personality Questionnaire with 100 items divided into three scales. By adding four new dimensions to the Questionnaire, the 240-yes/no-item Temperament and Character Inventory was devised. It is made up of seven scales divided into 25 subscales, including control scales for the assessment of the consistency of responses.

- Positive and Negative Syndrome Scale (PANSS) (Kay et al. 1986), (negative, positive and general psychopathology scales), with the aim to determine the severity of the current psychopathological problems.

- Brif psychiatric rating scale BPRS (Overall \& Gorham 1962) to confirm a diagnosis of the current psychopathological problems within psychotic disorders, consisting of 19 items, with 7 possible answers, and a scale 1-7 to indicate the severity of the symptoms.

- Sociodemographic data: gender (male, female), age, qualifications (elementary education which includes eight grades, secondary education which includes eight grades plus four grades of secondary school, and higher education which includes faculty after secondary education), employment (employed/ unemployed, pensioner), marital status (married, single, divorced, widowed), place of residence (countryside, city).

\section{Statistical analysis}

The sample size was determined using a calculator for the sample size calculations, and the pilot-study results were used as well. The pilot study showed that variables Novelty Seeking, Exploratory Excitability and Empathy make a difference between the patients with schizophrenia and the healthy controls. The calculator showed that the sample of healthy controls and the sample of patients should consist of at least 18 subjects, assuming an alpha error of 0.05 and the power level of the study 0.8 (beta error). Through binary logistic regression, the pilot study showed which variables could have an impact on the development of schizophrenia. Kolmogorov-Smirnov and Shapiro-Wilk tests were used to test the normal probability distribution of variables. Independent Samples T-Test and Mann-Whitney test were used to test the difference of mean values of variables between the two groups. Dependence of categorical variables was tested using the Chi-Square and Fisher's tests. The same methodological approach was used for making the mathematical model. The Model is the mathematical formula $p=e z /(1+e z)$, where $\mathrm{z}$ represents a sum which is calculated through variables whose impact is statistically significant for the development of the illness. This formula,for each patient, calculates the probability (or percentage, if the probability is multiplied by 100) that a subject suffers or 
will suffer from schizophrenia. The variable probability distribution to separate patients from healthy controls was tested using ROC curves. Data analyses relied on: frequencies, percentages, arithmetic means, standard deviations, the medians, the $25^{\text {th }}$ and the $75^{\text {th }}$ percentile and a $95 \%$ confidence interval. The obtained data were processed by descriptive statistics, stating the mean values. Values $\mathrm{p}<0.05$ were regarded as statistically significant.

\section{RESULTS}

Table 1. shows socio-demographic characteristics of the study sample.

The differences between the patients and the healthy controls are significant regarding the following variables: Exploratory Excitability $(\mathrm{p}<0.0005)$, Impulsiveness $(p<0.0005)$, Harm Avoidance $(p<0.0005)$, Anticipatory Worry $(p<0.0005)$, Fear of Uncertainty $(p<0.0005)$, Shyness $(p<0.0005)$, Fatigability $(p<0.0005)$, Reward Dependence $(p<0.0005)$, Sentimentality $(p=0.022)$, Attachment $(p<0.0005)$, Dependence $(p=0.026)$, Persistence $(p<0.0005)$, Self - directedness $(p<0.0005)$, Responsibility $(p<0.0005)$, Purposefulness $(p<0.0005)$, Resourcefulness $(p<0.0005)$, Self-acceptance $(p<0.0005)$, Congruent Second Nature $(\mathrm{p}<0.0005)$, Cooperativeness $(p<0.0005)$, Social Acceptance $(p<0.0005)$, Empathy $(\mathrm{p}<0.0005), \quad$ Helpfulness $\quad(\mathrm{p}<0.0005), \quad$ Compassion $(p<0.0005)$, Pure heartedness $(p<0.0005)$, Self - transcendence $(p<0.0005)$, Self-forgetfulness $(p<0.0005)$, Transpersonal Identification $(\mathrm{p}=0.001)$, Spiritual Acceptance $(\mathrm{p}=0.002)$. The data are shown in Table 2 .

Table 1. Sociodemographic characteristics

\begin{tabular}{lcc}
\hline & Healthy controls & Patients \\
\hline Sex & & \\
$\quad$ Males & $35(46.1 \%)$ & $48(63.1 \%)$ \\
Females & $41(53.9 \%)$ & $28(36.8 \%)$ \\
Education & & \\
$\quad$ Primary & $0(0.0 \%)$ & $18(23.7 \%)$ \\
Secondary & $22(28.9 \%)$ & $49(64.5 \%)$ \\
Higher & $54(871.1 \%)$ & $9(11.8 \%)$ \\
Marital status & & \\
Single & $35(46.1 \%)$ & $59(77.6 \%)$ \\
Married & $37(48.7 \%)$ & $9(11.8 \%)$ \\
Divorced & $4(5.3 \%)$ & $8(10.5 \%)$ \\
Employment & & \\
Unemployed & $11(14.5 \%)$ & $67(88.2 \%)$ \\
Employed & $65(85.5 \%)$ & $7(9.2 \%)$ \\
Pensioners & $0(0.0 \%)$ & $2(2.6 \%)$ \\
Residence & & \\
City & $65(85.5 \%)$ & $46(60.5 \%)$ \\
Countryside & $11(14.5 \%)$ & $30(39.55 \%)$ \\
\hline
\end{tabular}

Table 2. Comparison of the TPQ parameters between the patients and the healthy controls

\begin{tabular}{|c|c|c|c|c|}
\hline & Healthy controls & Patients & $P$ & Healthy controls \\
\hline \multirow[t]{2}{*}{ Novelty Seeking } & Exploratory Excitability & $49.00(44.00-53.00)$ & $44.00(36.00-49.00)$ & $<0.0005$ \\
\hline & Impulsiveness & $42.00(38.00-51.00)$ & $56.00(47.00-60.00)$ & $<0.0005$ \\
\hline \multirow[t]{5}{*}{ Harm Avoidance } & Harm Avoidance & $49.00(43.00-55.50)$ & $61.50(52.00-70.50)$ & $<0.0005$ \\
\hline & Anticipatory Worry & $49.00(45.00-57.00)$ & $62.00(53.00-70.00)$ & $<0.0005$ \\
\hline & Fear of Uncertainty & $52.00(44.50-57.00)$ & $57.00(52.00-62.00)$ & $<0.0005$ \\
\hline & Shyness & $49.00(43.00-57.00)$ & $57.00(49.00-64.00)$ & $<0.0005$ \\
\hline & Fatigability & $52.00(44.00-56.00)$ & $61.00(52.00-70.00)$ & $<0.0005$ \\
\hline Reward of & Reward Dependence & $51.00(44.00-56.00)$ & $44.00(39.00-49.00)$ & $<0.0005$ \\
\hline \multirow[t]{3}{*}{ Dependence } & Sentimentality & $44.00(39.00-53.00)$ & $44.00(39.00-53.00)$ & 0.022 \\
\hline & Attachment & $56.00(47.00-56.00)$ & $47.00(38.00-56.00)$ & $<0.0005$ \\
\hline & Dependence & $47.00(41.00-53.00)$ & $47.00(41.00-47.00)$ & 0.026 \\
\hline Persistence & Persistence & $47.00(39.00-52.00)$ & $36.00(31.00-49.50)$ & $<0.0005$ \\
\hline \multirow[t]{6}{*}{ Self Directedness } & Self - Directedness & $53.50(46.00-58.00)$ & $36.00(30.00-45.00)$ & $<0.0005$ \\
\hline & Responsibility & $56.00(46.00-61.00)$ & $36.00(26.00-43.50)$ & $<0.0005$ \\
\hline & Purposefulness & $53.00(47.00-58.00)$ & $44.50(36.50-46.00)$ & $<0.0005$ \\
\hline & Resourcefulness & $50.00(42.00-50.00)$ & $33.00(21.00-42.00)$ & $<0.0005$ \\
\hline & Self Acceptance & $56.00(45.00-63.00)$ & $47.00(41.00-52.00)$ & 0.002 \\
\hline & Congruent $2^{\text {nd }}$ Nature & $54.00(46.00-58.00)$ & $42.00(36.00-46.00)$ & $<0.0005$ \\
\hline \multirow[t]{6}{*}{ Cooperativeness } & Cooperativeness & $52.00(48.00-55.00)$ & $41.00(34.00-45.00)$ & $<0.0005$ \\
\hline & Social Acceptance & $52.00(45.00-59.00)$ & $39.00(25.00-45.00)$ & $<0.0005$ \\
\hline & Empathy & $51.50(41.00-55.00)$ & $34.00(26.00-41.50)$ & $<0.0005$ \\
\hline & Helpfulness & $48.00(42.00-48.00)$ & $36.00(36.00-42.00)$ & $<0.0005$ \\
\hline & Compassion & $55.00(51.00-59.00)$ & $51.00(37.00-55.00)$ & $<0.0005$ \\
\hline & Pure - heartedness & $52.00(48.00-57.00)$ & $48.00(43.00-52.00)$ & $<0.0005$ \\
\hline \multirow[t]{4}{*}{ Self Transcendence } & Self Transcendence & $39.00(32.00-47.00)$ & $39.00(32.00-47.00)$ & $<0.0005$ \\
\hline & Self - forgetfulness & $39.00(36.00-47.00)$ & $54.00(43.00-61.00)$ & $<0.0005$ \\
\hline & Transpersonal Identification & $43.00(39.00-52.00)$ & $52.00(43.00-60.00)$ & 0.001 \\
\hline & Spiritual Acceptance & $37.00(30.00-48.00)$ & $48.00(34.00-58.00)$ & 0.002 \\
\hline
\end{tabular}

Values are presented as the medians (the $25^{\text {th }}$ percentile - the $75^{\text {th }}$ percentile) 
Table 3. Sample for model and sample for testing

\begin{tabular}{lccc}
\hline & Sample for model & Sample for testing & $\mathrm{P}$ \\
\hline Age & $36.95 \pm 9.776$ & $36.20 \pm 9.342$ & 0.652 \\
Number of hospitalizations & $3.00(1.00-7.00)$ & $3.00(1.00-500)$ & 0.595 \\
Duration of illness & $11.00(5.50-16.00)$ & $10.00(3.00-15.00)$ & 0.212 \\
\hline
\end{tabular}

Table 4. Binary logistic regression

\begin{tabular}{|c|c|c|c|c|c|}
\hline & \multicolumn{2}{|c|}{ Univariate binary logistic regression } & \multicolumn{2}{|c|}{ Multivariate binary logistic regression } \\
\hline & & Odds ratio & $\mathrm{P}$ & Odds ratio & $\mathrm{P}$ \\
\hline \multirow{7}{*}{$\begin{array}{l}\text { Novelty } \\
\text { Seeking } \\
\text { Harm } \\
\text { avoidance }\end{array}$} & Exploratory Excitability & $0.909(0.863-0.959)$ & $<0.0005$ & & \\
\hline & Impulsiveness & $1.063(1.023-1.106)$ & 0.002 & & \\
\hline & Harm avoidance & $1.135(1.077-1.197)$ & $<0.0005$ & & \\
\hline & Anticipatory Worry & $1.100(1.046-1.159)$ & $<0.0005$ & & \\
\hline & Fear of Uncertainty & $1.101(1.050-1.141)$ & $<0.0005$ & & \\
\hline & Shyness & $1.131(1.070-1.195)$ & $<0.0005$ & & \\
\hline & Fatigability & $1.123(1.069-1.180)$ & $<0.0005$ & & \\
\hline \multirow{2}{*}{$\begin{array}{l}\text { Reward of } \\
\text { dependence }\end{array}$} & Reward of dependence & $0.916(0.873-0.960)$ & $<0.0005$ & & \\
\hline & Attachment & $0.876(0.827-0.927)$ & $<0.0005$ & & \\
\hline Persistance & Persistence & $0.940(0.902-0.979)$ & 0.003 & & \\
\hline \multirow{6}{*}{$\begin{array}{l}\text { Self } \\
\text { directedness }\end{array}$} & Self directedness & $0.871(0.827-0.919)$ & $<0.0005$ & & \\
\hline & Responsibility & $0.875(0.832-0.919)$ & $<0.0005$ & $0.903(0.834-0.976)$ & 0.011 \\
\hline & Purposefulness & $0.943(0.908-0.979)$ & 0.002 & $1.109(1.027-1.198)$ & 0.009 \\
\hline & Resourcefulness & $0.880(0.839-0.924)$ & $<0.0005$ & $0.909(0.847-0,976)$ & 0.009 \\
\hline & Self Acceptance & $0.958(0.920-0.997)$ & 0.036 & & \\
\hline & Congruent $2^{\text {nd }}$ Nature & $0.867(0.818-0.919)$ & $<0.0005$ & & \\
\hline \multirow{6}{*}{$\begin{array}{l}\text { Cooperative } \\
\text { ness }\end{array}$} & Cooperativeness & $0.841(0.786-0.991)$ & $<0.0005$ & $0.773(0.661-0.904)$ & 0.001 \\
\hline & Social Acceptance & $0.905(0.864-0.931)$ & $<0.0005$ & & \\
\hline & Empathy & $0.893(0.854-0.935)$ & $<0.0005$ & & \\
\hline & Helpfulness & $0.847(0.786-0.913)$ & $<0.0005$ & & \\
\hline & Compassion & $0.908(0.860-0.959)$ & $<0.0005$ & $1.166(1.007-1.349)$ & 0.040 \\
\hline & Pure heartedness & $0.893(0.835-0.955)$ & 0.001 & & \\
\hline \multirow{4}{*}{$\begin{array}{l}\text { Self trans- } \\
\text { cendence }\end{array}$} & Self transcendence & $1.055(1.020-1.091)$ & 0.002 & & \\
\hline & Self forgetfulness & $1.082(1.037-1.128)$ & $<0.0005$ & & \\
\hline & Transpersonal Identification & $1.056(1.013-1.100)$ & 0.010 & & \\
\hline & Spiritual Acceptance & $1.034(1.03-1.065)$ & 0.032 & & \\
\hline
\end{tabular}

A sample of 102 participants was used to make the Model. We then tested the Model on another 50 participants. The difference between the study groups for making and testing the Model in mean values of age $(\mathrm{p}=0.652)$, number of hospitalizations $(\mathrm{p}=0.595)$ and duration of disorder $(\mathrm{p}=0.212)$ was not statistically significant. There was no connection between the study groups for making and testing the Model on one hand, and gender $(\mathrm{p}=1.000)$, qualifications $(\mathrm{p}=0.944)$, employment rate $(\mathrm{p}=0.862)$ and place of residence $(\mathrm{p}=0.996)$ on the other. These tests showed that the samples for making and testing the Model were similar. The data are shown in Table 3.

Univariate binary logistic regression indicated that the development of schizophrenia is affected by the following temperament dimensions: Novelty Seeking NS (Exploratory Excitability, Impulsiveness), Harm Avoidance - HA (Anticipatory Worry, Fear of Uncertainty, Shyness, Fatigability), Reward of Dependence RD (Attachment), Persistence - P, as well as: Self Directedness SD (Responsibility, Purposefulness, Resourcefulness, Self-acceptance, Congruent $2^{\text {nd }}$ Nature),
Cooperativeness - C (Social Acceptance, Empathy, Helpfulness, Compassion, Pure heartedness), Self Transcendence - ST (Self Forgetfulness, Transpersonal Identification, Spiritual Acceptance).

Multivariate binary logistic regression showed that Responsibility, Purposefulness, Resourcefulness, Cooperativeness and Compassion are statictically more manifested and affect the development of schizophrenia. See Table 4.

Multivariate binary logistic regression was used for making the Model. Wald's Backward Method was used as well. The coefficients of multivariate binary logistic regression are shown in Table 5.

Table 5. Coefficients of binary regression

\begin{tabular}{lc}
\hline & Coefficients \\
\hline Responsibility & -0.102 \\
Purposefulness & 0.103 \\
Resourcefulness & -0.095 \\
Cooperativeness & -0.257 \\
Compassion & 0.153 \\
Constant & 7.339 \\
\hline
\end{tabular}


The Model represents a new variable devised in the following way:

$$
\begin{aligned}
& \text { Model } 1=100 \cdot \mathrm{e}^{\text {sum }}\left(1+\mathrm{e}^{\text {sum }}\right) \text {, where } \\
& \text { Sum }=-0.102 \cdot \text { Ry }+0.103 \cdot P-0.095 \cdot R s-0.257 \cdot C s+0.153 \cdot C n+7.339 \text {. } \\
& \text { Ry }- \text { Responsibility; P }- \text { Purposefulness; Rs --Resourcefulness; } \\
& \text { Cs -.Cooperativness; Cn -.Compassion }
\end{aligned}
$$

The value of the Model was calculated for each database patient, showing a probability distribution for developing schizophrenia. For instance, the value of the Model for patient number 31 (from the test group) was $98.44 \%$, showing that he developed schizophrenia, where as for patient number 37 (also from the test group) it was $4.34 \%$, meaning he did not develop schizophrenia.

A sample of 50 participants was recruited to test the Model. The ROC curve of this sample (see Figure 1) shows that the variable Model represents a reliable marker for identifying people at high risk of developing schizophrenia (area $=0.896, \mathrm{p}<0.0005$ ). The cut-off score was 59 . The Model yielded a sensitivity of $80 \%$ and a specificity of $84 \%$. As a result, there were $80 \%$ of patients who exhibited the value of the variable Model higher than 59 and $84 \%$ of healthy controls less than or equal to 59 respectively.

Table 6 shows the ratio between the patients and healthy controls screened for positive and negative predictive values of the Model.

Table 6. True and false positive and negative

\begin{tabular}{llcc}
\hline Symbol & $\begin{array}{c}\text { Healthy } \\
\text { controls }\end{array}$ & Patients \\
\hline Model & Positive (Model 1 $\geq 59$ ) & 4 & 20 \\
& Negative (Model 1 <59) & 21 & 5 \\
\hline
\end{tabular}

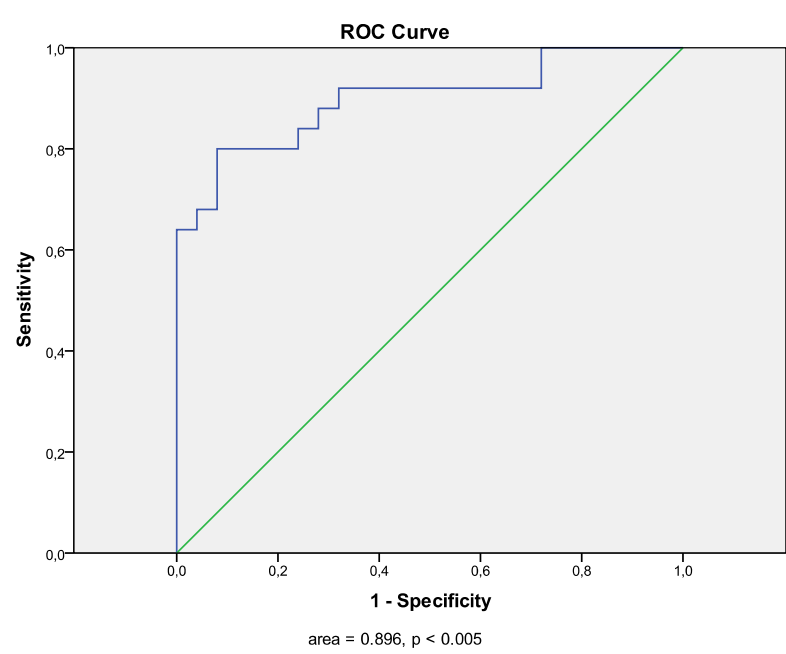

Figure 1. ROC curve for the Model

\section{DISCUSSION}

We used Cloninger's Temperament and Character Inventory (TCI) to test personality traits that have a biological foundation. Unlike healthy controls, we found that patients with schizophrenia exhibited higher
Harm Avoidance scores, not uncommon for people who are constantly worried, pessimistic, afraid and shy, which is consistent with previous studies (Guillem et al. 2002; Jetha et al. 2013). Likewise, the Self-transcendence dimension showed high scores. This dimension may be expessed in high spirituality and creativity, and on the other hand it may leave the patients in an autistic and closed world, which may further lead to psychotic fantasies (Song et al. 2013; Lee et al. 2016). Patients exhibited lower Self-directedness (SD) scores compared to the healthy controls, characterizing them as immature, fragile, unreliable, with no long-term goals. They had the same Cooperativeness (C) scoring, which shows that they are socially intolerant, critical and unwilling to provide any sort of help. The findings coincide with previous studies (Guillem et al. 2002). The same results were obtained in the study on Japanese population (Ohi et al. 2012). According to Cloninger, persons with lower Self-directedness, the patients in our study, are less liable, less integrated, have a lower self-esteem and are less adapted (Cloninger et al. 1994). Self-directedness (SD) is typically low in the patients with schizophrenia (Smith et al. 2008). The patients with schizophrenia in this study exhibited significantly lower resultsat the Reward Dependence (RD). Cloninger interprets this low result through the distancing, weaker social sensitivity and communicativity. Cloninger's factor model is successfully used in other studies (Saulsman et al. 2004). Studies dealing with the same topic have, in addition to the patients with schizophrenia and healthy controls, included the patients with a schizoaffective disorder or with a schizotypical personality disorder as well. Some of the studies have used the results corrected by gender for each personality trait. All this has led to a heterogeneity in the results, and in order to avoid it, the subgroup analysis that excludes these studies from a meta analysis has shown that their results have not affected the overall results (Ohi et al. 2012; GonzalesTorres et al. 2009; Cortes et al. 2009). Generally speaking, certain personality traits may have a protective or vulnerable role in developing schizophrenia. Personality traits measured by the TCI questionnaire among the general population vary between the cultures (Pelissolo \& Lepine2000, Brandstrom et al. 2001), so that they may affect the study results. In the study conducted by Ohi et al, there was found the intercultural difference in NS that exists in the Asian population between the patients with schizophrenia and the control group, which was not found in the European population (Ohi et al. 2012).

Through the multivariate binary logistic regression, the following variables were selected: Responsibility, Purposefulness, Resourcefulness, Cooperativeness and Compassion. They are statistically significant and have an impact on the onset of the disease. This means that higher values of Responsibility, Resourcefulness and Cooperativeness decrease the risk of developing schizophrenia, while higher values of Purposefulness and Shyness increase the risk. The lower the value of 
Responsibility and Cooperativeness, the higher the risk of developing schizophrenia. It is often difficult to detect whether a person is ill. It would be good to create a simple test, which would be applicable in practice and based on which it would be possible to give an assessment. Assume that there is such a test and that it is given to one group of subjects. After that, it would be clear who suffers from certain illnesses, who does not suffer from any or who is at risk of developing an illness. The test results might be positive (indicating the presence of an illness) or negative (indicating the absence of an illness) and do not have to comply with the diagnosis. The question is if any TPQ variable may (by itself) be a marker for the illness. Binary logistic regression, on the basis of five variables with a statistically significant influence on the manifestation of the illness, through the mathematical formula, helped us create the model. The importance of this research is in creating such a model. For example, we do not know whether a subject suffers from schizophrenia. In case that our model shows, for example, that the odds that a subject suffers from or might develop schizophrenia are $42 \%$, such subject requires further attention. If the odds are, for example, $0.9 \%$, there is a small possibility that a subject suffers or is likely to develop schizophrenia. The Model is applicable in practice, which has been confirmed through our research, since it represents a good marker in separating the healthy and the ill. It can be modified on a larger sample as well, which would require further research. It might be applied in primary healthcare, primarily through the Community Mental Health Centres, for young persons with a positive family heredity.

Our Study has limitations as well. The size of the sample is relatively small for the complexity ofthe research variables. Such size of the sample may affect the data processing. This particularly refers to the multivariate statistical procedures carried out on a smaller sample. Another limitation is the fact that the data were collected through a questionnaire filled in by the interviewed persons themselves, whereby the filling of the questionnaire may be modified by various levels of the patients' fatigue and motivation.

\section{CONCLUSIONS}

Our research confirmed the differences in temperament (HA) and character (SD, ST and C) between the patients with schizophrenia in the remission phase and the healthy controls, which coincides with the most of the related studies. Based on the Temperament and Character Inventory, five parameters were taken to make the mathematical model. The Model is a new variable and we consider it would be a reliable marker for identifying young people at high risk of developing schizophrenia (due to its good sensitivity and specificity). We also consider that the Model should be applied in primary healthcare, i.e. in the Community Mental Health Centres, primarily for the risk assessment.

\section{Acknowledgements: None.}

\section{Conflict of interest: None to declare.}

\section{Contribution of individual authors:}

Mirjana Mišković is the author of the Study, who has designed the Study, developed the idea and its form, the methods of conducting the Study, obtaining and collecting the data, conducting statistical analysis and interpretation of the data. She has prepared the first draft and has critically corrected the paper.

Dragan Ravanić is a co-author, who has contributed to the idea and form, the methods of performing the Study and critical correction of the paper.

Banković Dragić is a co-author, who has contributed to the analysis and interpretation of the data.

Nera Zivlak Radulović is a co-author, who has contributed to obtaining and collecting the data, as well as to writing the paper.

Višnja Banjac is a co-author, who has contributed to obtaining and collecting the data, as well as to writing the paper.

Tatjana Dragišić is a co-author, who has contributed to the analysis and interpretation of the data.

\section{References}

1. Aukst Margetic B, Jakovljevic M, Brataljenovic $T$ \& Šumic M: Personality and schizophrenia: Psychobiological model and its relationship with comorbidity. Psychiatr Danub 2009; 21:356-360

2. Brandstrom S, Richter J, Przybeck T: Distributions by age and sex of the dimensionsof temperament and character inventory in a cross-cultural perspective among Sweden, Germany and the USA. Psychological Reports 2001; 89:747-758

3. Cloninger CR, Svrakic DM \& Przybeck TR: A psychobiological model of temperament and character. Archives of General Psychiatry 1993; 50:975-990

4. Cloninger $R$, Przybeck T, Svrakic D, Wetzel R: The temperament and character inventory. A guide to its Development and use. Center for psychobiology of personality 1994, Washington University, St.Louis Missouri

5. Cloninger CR: A systematic method for clinical description and classification of personality variants. A proposal. Archives of General Psychiatry 1987; 44:573-588

6. Cortes MJ, Valero J, Gutierrez-Zotes JA, Hernandez A, Moreno L, Jariod M, Martorell L, Vilella E, Labad A: Psychopathology and personality traits in psychotic patients and their first-degree relatives. European Psychiatry 2009; 24:476-482

7. Gonzalez-Torres MA, Inchausti L, Ibanez B, Aristegui M, Fernandez-Rivas A, Ruiz E, Fernandez E, Bayon C: Temperament and character dimensions in patients with schizophrenia, relatives, and controls. The Journal of Nervous and Mental Disease 2009; 197:514-519

8. Guillem F, Bicu M, Semkovska M, \& Debruille JB: The dimensional symptom structure of schizophrenia and its association with temperament and character. Schizophrenia Research 2002; 56: 137-147 
9. Hori H, Noguchi H, Hashimoto R, Nakabayashi T, Saitoh $O$, Murray RM, Okabe S, Kunugi H: Personality in schizophrenia assessed with the Temperament and Character Inventory (TCI). Psychiatry Research 2008; 160:75-183

10. Jetha MK, Goldberg JO and Schmidt LA: Temperament and its relation to social functioning in schizophrenia. Int J Soc Psychiatry 2013; 59:254

11. Kay SR, Opler LA, Fiszbein A: Positive and Negative Syndrome Scale (PANSS) Manual. North Tonawanda, NY, Multi-Health Systems 1986

12. Kurs R, Farkas H, \& Ritsner M: Quality of life and temperament factors in schizophrenia: Comparative study of patients, their siblings and controls. Quality of Life Research 2005; 14: 433-440

13. Lee DB, Park MJ, Lee MY, Moon E, Jeong JH, Chung IY, Young MY: Heritability and Familiality of Temperament and Character Dimensions in Korean Families with Schizophrenic Linkage Disequilibrium. Clin Psychopharmacol Neurosci 2016; 14:203-209

14. Ohi K, Hashimoto R, Yasuda Y, Fukumoto M, Yamamori H, Iwase M, Kazui H, Takeda M: Personality traits and schizophrenia: evidence from a case-control study and meta-analysis. Psychiatry Res 2012, 198:7-11

15. Ostojic D: The first episode of schizophrenia - the importance of early detection of illness. What do social workers need to know? Social work Annual 2012; 19:53-72

16. Overall JE, Gorham DR: The Brief Psychiatric Rating Scale. Psychol Rep 1962; 10:799-812
17. Pelissolo A, Lepine JP: Normative data and factor structure of the Temperament andCharacter Inventory (TCI) in the French version. Psychiatry Research 2000; 94:67-76

18. Popovic I: Analysis of the factors that contribute to the development of metabolic syndrome in long-term hospitalized schizophrenic patients treated with atypical antipsychotics. Doctoral thesis 2014, Kragujevac

19. Ritsner M, Farkas $H$, \& Gibel A: Satisfaction with quality of life varies with temperament types of patients with schizophrenia. Journal of Nervous and Mental Disease 2003; 191:668-674

20. Saulsman LM, Page AC: The five-factor model and personality disorder empirical literature: A meta-analytic review. Clinical Psychology Review 2004; 23:1055-1085

21. Schenkel LS, Silverstein SM: Dimensions of premorbid functioning in schizophrenia: a review of neuromotor, cognitive, social, and behavioral domains. Genetic, Social, and General Psychol, Monogr 2004; 130:241-270

22. Smith MJ, Cloninger CR, Harms MP, Csernansky JG: Temperament and character as schizophrenia-related endophenotypes in non-psychotic siblings. Schizophrenia Research 2008; 104: 198-205

23. Song YY, Kang JI, Kim SJ, Lee MK, Lee E, An SK: Temperament and character in individuals at ultra-high risk for psychosis and with first-episode schizophrenia: associations with psychopathology, psychosocial functioning, and aspects of psychological health. Compr Psychiatry 2013; 54:1161-1168

Correspondence:

Mirjana Miskovic, MD, MsC, Psychiatrist

Psychiatric Clinic, University Clinical Center of the Republic of Srpska

F.G. Lorke 5, 78000 Banjaluka, Bosnia and Herzegovina

E-mail: miskovicmirjana@yahoo.com 